This PDF is a selection from an out-of-print volume from the National Bureau of Economic Research

Volume Title: Frontiers in Health Policy Research, Volume 3

Volume Author/Editor: Alan M. Garber, editor

Volume Publisher: MIT Press

Volume ISBN: 0-262-57141-2

Volume URL: http://www.nber.org/books/garb00-1

Publication Date: January 2000

Chapter Title: Comparing the Quality of Health Care Providers

Chapter Author: Mark McClellan, Douglas Staiger

Chapter URL: http://www.nber.org/chapters/c9832

Chapter pages in book: (p. 113 - 136) 


\title{
Comparing the Quality of Health Care Providers
}

\author{
Mark McClellan, Stanford University and the NBER \\ Douglas Staiger, Dartmouth College and the NBER
}

\section{Executive Summary}

This article introduces a new approach for evaluating the quality of health care providers, including an integrated solution to several problems that limit the usefulness of available methods. Our approach combines information from all the quality indicators available for a provider (e.g., from other years, other patients, or other indicators for the same patients) to estimate more accurately the provider's previous or expected quality. The approach also provides an empirical basis for comparing and combining alternative quality indicators, thereby enabling policy makers to choose among potential indicators, explore the potential bias in alternative measures, and increase the value of quality measures for assessing and improving care. Using hospital care for elderly heart attack patients as an example, we demonstrate that these methods can be used to create reliable, precise predictions about provider quality. Comparing quality of care across providers may be far more feasible than many now believe.

\section{Introduction}

Comparing quality of care across providers is becoming increasingly important, both as a contracting and quality improvement tool for health plans and payers, and as "report cards" to help consumers and others compare quality of care. Examples include rankings of the "best" hospitals published by magazines such as US News $\&$ World Report, scorecards issued by state regulators in Pennsylvania and New York, and hospital quality reports produced by the Health Care Financing Administration (HCFA) and the Agency for Healthcare Research and Quality (AHRQ).

Despite enormous interest in developing such quality assessment tools in health care, these measures have generated much controversy and skepticism. The problem with many of these rankings is that they simply are not viewed as particularly informative (Hofer et al. 1999; 
Iezzoni 1997). This perception is especially true for measures based on important patient outcomes, which tend to be imprecise and particularly susceptible to differences in patient severity and comorbidity because of the relatively small samples of patients seen by most providers in a single year. In particular, providers identified as providing high quality care in one year often appear to provide low quality care in the subsequent year. For example, table 5.1 shows how hospitals identified in 1990 as the "best" (top 10 percent) hospitals on one common quality indicator, thirty-day mortality rate following a heart attack, were ranked in subsequent years (this data is described in more detail below). Hospitals that were identified as the "best" in 1990 were nearly as likely to be ranked among the worst (14.7 percent) as among the best (16.7 percent) by 1994. In fact, average mortality rates among these hospitals were actually higher than average by 1994 . This type of evidence has led many to wonder whether rankings based on such data do more harm than good (Bindman 1999).

Most of the existing provider report cards have responded to these perceived problems by moving away from an emphasis on serious outcomes of important health problems. For example, HEDIS measures have emphasized screening and relatively common process measures of care. Other evaluators have focused on larger hospitals or groups of physicians. Others have argued for expensive collection of detailed case-mix measures to reduce noise and bias. Still others have advocated collecting large numbers of measures and aggregating them according to a priori rules.

These perceived problems with provider report cards have resulted in considerable debate over the uses of such information. But given the growing pressures placed on health care providers to provide efficient high quality care, the trend toward greater accountability will continue-with increasing pressure to develop and apply measures to finer levels of analysis (e.g., individual physicians, particular groups of patients). Thus, the fundamental question is, can we develop better methods for comparing quality across providers in such situations?

In a series of recent papers (McClellan and Staiger 1999a, 1999b; Geppert, McClellan, and Staiger 2000), we have developed methods that address these concerns and have applied our approach in ongoing work to implement better quality evaluation and improvement methods for private health plans, health care purchasing groups, and Medicare. We refer to our new quality indicators as "filtered" estimates of provider quality because one of the key advantages of these indica- 
Table 5.1

Comparisons of hospital rankings: 1990, 1992, and 1994

Ranked in best $10 \%$

(lowest mortality rate)

in 1990

Of the hospitals ranked in the best $10 \%$ in 1990 :

1992

What percentage were still ranked in the best

$17.0 \%$

$10 \%$ (lowest mortality rates)?

What percentage were ranked in the worst

$10 \%$ (highest mortality rates)?

Average difference in mortality rate from all

other hospitals (standard error of estimate)

(.005)

1994

What percent were still ranked in the best

$10 \%$ (lowest mortality rates)?

What percent were ranked in the worst $10 \%$ (highest mortality rates)?

Average difference in mortality rate from all

other hospitals (standard error of estimate)

$10.4 \%$

$-.014$

$(005)$

$16.7 \%$

$14.7 \%$

$+.005$

(.006)

Note: Rankings based on conventional profiling methods. Outcome measure: risk-adjusted thirty-day AMI mortality among the elderly.

tors is that they filter out the estimation error that plagues conventional quality indicators and that leads to the type of instability seen in table 5.1. By integrating these methods with measures of important processes and outcomes of care, we can develop more precise, comprehensive, and informative quality evaluations than have previously been possible. This article provides an overview of the method and illustrates its application in evaluating the quality of hospital care for elderly heart attack patients. Readers interested in the technical details, along with a more comprehensive empirical evaluation of the performance of our approach, are referred to our earlier articles, particularly McClellan and Staiger (1999a).

Our method combines information from all the quality indicators available for a provider (e.g., from other years, other patients, or other indicators for the same patients) to estimate more precisely the provider's current or future quality level. The basic idea is simple. Any single quality indicator for a provider will be a very imprecise, that is, "noisy" measure of that provider's quality. But the array of quality indicators that we observe for a provider is likely to be related across different quality measures and over time. Therefore, the core idea of our 
method is to use these relationships between multiple measures and over time to extract a more accurate "signal" in each particular measure. In addition to improving the precision of the estimated quality indicators, our method provides an empirical basis for comparing alternative quality indicators and exploring potential biases. This approach enables one to choose among potential indicators and, in many cases, limit the number of quality indicators either collected or reported.

Our particular application of these methods illustrates many of the challenges facing quality evaluation in health care. On one hand, heart disease is the leading cause of death in the United States and is clearly a condition for which the quality of medical care may have a substantial impact on an individual's health. On the other hand, most hospitals treat relatively few heart attack patients in any given year, and conventional quality indicators of the most important outcomes, such as survival, are imprecise because of the influence of many factors besides hospital quality. We use this example to demonstrate how our filtering methods can be used to create reliable, precise predictions about provider quality. In the conclusion, we discuss additional promising applications of our work to improving health care quality.

\section{Uses and Limitations of Conventional Quality Indicators}

\section{Uses of Quality Indicators}

Comparing quality of care across providers is an increasingly important tool as the health care market is becoming more competitive. The Institute of Medicine (1990) has defined quality as "the degree to which health services for individuals and populations increase the likelihood of desired health outcomes and are consistent with current professional knowledge." But to translate this general definition into concrete quality indicators, one must ask how the indicators will be used and consider the features that are required of a quality indicator in that use.

Why do we measure quality? Ultimately, the goals are to help guide decision making and improve quality of care. To achieve these goals, information on provider quality is used primarily in three ways: for contracting with providers, for guiding consumers in making informed choices among providers, and for identifying exemplary providers to serve as models of best practice.

Quality information is used in contracts between purchasers and providers as a way of providing incentives to the provider to maintain 
quality of care. Such incentives have become an increasingly important safeguard because purchasers have moved toward capitation and other fixed-price contracts that place financial pressure on providers to reduce the cost of care. This use of quality information is largely backward looking, for example, basing financial rewards on whether the provider achieved concrete and quantifiable quality standards over the previous year.

Quality information is also used to guide consumers (and other purchasers such as employers and health plans) in making informed choices among providers. In today's health care market, providers are increasingly competing for patients on the basis of price, which in turn increases the incentives for providers to reduce the amount of care given to patients. Not surprisingly, lack of accurate quality information can result in reduced quality in a competitive market (Dranove and Satterthwaite 1992). Therefore, providing purchasers with accurate quality information so that they can make informed trade-offs between cost and quality is a cornerstone of competitive approaches (Enthoven and Kronick 1989). This use of quality information is largely forward looking, trying to forecast current or future quality based on available data from the past.

Finally, quality information is used to identify exemplary providers who serve as models of best practice (or to identify low-quality providers for some form of intervention). This approach is a management tool used to encourage the adoption of best practice as part of many quality improvement initiatives. This use of quality information also tends to be forward looking, using data from the past to identify providers for which the quality of care is currently likely to be particularly good or bad.

\section{Limitations of Conventional Quality Indicators}

A key obstacle to using quality indicators in any of these ways is the substantial amount of imprecision associated with almost all important measures of provider quality. Most serious health problems, such as heart attacks, are relatively infrequent; many hospitals may treat only a few dozen patients or fewer over an entire year. And most major outcomes, such as long-term mortality, will be influenced by an enormous number of factors other than the quality of the provider. Therefore, it is almost impossible to assess any single outcome measure for a particular provider with any degree of precision. As a result, these measures are often poor indicators of past and future performance. 
This lack of precision in conventional quality indicators limits their practical value. Contracts that reward providers based on an imprecise indicator provide weak incentives (because the reward is only weakly related to actual quality of care) while exposing providers to unnecessary financial risk. Inaccuracy can also lead to a lack of trust in the quality indicators, leading both purchasers and health providers to ignore the measures in decision making (Bindman 1999; Schneider and Epstein 1998). Finally, inaccuracy in conventional quality indicators will lead to a mislabeling of many ordinary providers as "exemplary" (as was suggested in table 5.1), thereby limiting our ability to learn from the experience of successful providers.

The natural response to this lack of precision is to refocus attention on measures for which the noise problem is less of an issue: health plans or medical groups rather than individual physicians, large teaching hospitals rather than all hospitals in a community, or preventive care and common illnesses rather than more serious illness. For example, the National Committee for Quality Assurance (NCQA), one of the leading organizations in the development of quality assessment measures, has focused on developing measures for which more than 400 cases per sample can reasonably be collected in a specific time period (e.g., six months or a year). This approach rules out measures for small providers (e.g., physicians) and for nearly all conditions serious enough to require hospital care.

This push toward more aggregation moves us in the wrong direction in terms of how consumers, purchasers, and health plans would like to use quality measures. Such aggregate measures provide poorly targeted incentives and are at best crude guides for identifying "exemplary" providers. By excluding many providers from consideration, we reduce the relevance of the measures for guiding decisions-especially because quality problems are often greater for lower-volume providers.

Another response to the lack of precision in conventional quality indicators has been to collect and report information on a large number of indicators in the hope that this will overcome the limitations of any one indicator. Most provider report cards report information on several quality indicators, including measures of patient satisfaction, preventive care, process of care, and patient outcomes such as death or important complications. For example, the California Healthcare Foundation medical group report card (QA Tool) has almost 1,100 measures.

The multidimensionality of current quality information raises two important concerns. First, the cost of collecting and processing this data 
is significant (Medicine $\mathcal{E}$ Health 1997). Some measures, such as long-term mortality, require costly follow-up and necessarily involve long reporting delays. Other measures require detailed chart review, which can be even more costly. Thus, from a practical perspective, we would like to target scarce resources on a few core indicators and eliminate redundant or otherwise unnecessary measures, particularly those that require additional costly data collection or that lead to delay. A second problem with the multidimensionality of current quality report cards is that the complexity makes them difficult to interpret. One expects that many of the measures are capturing a similar dimension of quality (e.g., surgical skill or quality of the staff in a particular specialty such as obstetrics). Thus, a systematic method that reduces many closely related measures into one, or identifies the one measure that best summarizes this dimension, would be valuable.

A final response to the perceived lack of reliability of conventional quality indicators has been to collect more detailed information on patient condition (severity and comorbidity), often from patient charts, to control more effectively for differences in patient condition across providers. The debate has been extensive over the potential bias in conventional quality indicators because of not controlling carefully for patient condition, but the empirical evidence for the existence of bias is mixed (Landon et al. 1996; Krakauer et al. 1992; Park et al. 1990). This debate is critical to the future of quality assessment because the high cost of gathering detailed information on patient condition can make quality assessment infeasible in the long term. For example, in a recent attempt to investigate this issue, HCFA collected chart information on all Medicare hospital admissions for cardiac conditions in 1994-1995, but the cost was over $\$ 100$ per case (Jeffrey Newman, personal communication, 1998). Obviously, it would be useful to have a systematic method for determining whether the additional expense of such measures was necessary.

\section{A Brief Overview of the Filtering Method}

Past work comparing quality of care in hospitals has generally relied on a single hospital outcome measure in a given year. For example, to compare mortality rates at two hospitals, one would simply calculate the average mortality rate at each hospital, generally adjusting for differences across hospitals in patient demographics and comorbidities, and assess whether the difference in this risk-adjusted mortality rate between the two hospitals was statistically significant. The limitation 
of this approach is that the estimates of mortality rates at any given hospital are generally imprecise because of the typically small samples of patients treated at a given hospital. For example, in a sample with 100 patients in each hospital, this approach generally cannot detect a significant difference if one hospital is estimated to have a 16 percent mortality rate and the other a 24 percent mortality rate. Typically, quality indicators are based on even smaller samples and the imprecision is even greater.

As an alternative, one can combine information from all the outcome measures available for a given hospital (e.g., other years, other patients, other outcomes for the same patients) to estimate more precisely a hospital's current quality. This approach is taken by McClellan and Staiger (1999a). We provide a nontechnical overview of the method below.

Suppose we observe a vector of conventional quality indicators $\left(M_{i}\right)$ for each hospital ( $i$ ). Each quality indicator is an estimate of the average of some variable thought to be related to quality of care, based on a sample of patients treated at that hospital over a specified period of time (for example, one year). Thus, $M_{i}$ might include measures of risk-adjusted mortality rates, average length of stay, rates of certain treatments, complication rates, or patient satisfaction levels. Each of these measures may be available for a range of patient samples, such as heart attack patients, very low birthweight infants, or those differentiated by insurance coverage. In addition, $M_{i}$ might include past years of information on many of these measures. In other words, one can think of $M_{i}$ as potentially including all of the conventional quality indicators that have ever been collected for a given hospital.

Conventional quality indicators are based on a sample of patients and are therefore imprecise estimates of what we really wish to measure. In any given year, even a high quality hospital may have poor patient outcomes because of chance events. This possibility is particularly likely when a quality indicator is based on only a handful of patients, so that the outcome of one or two patients will materially influence that hospital's estimate. Thus, we can think of the observed vector of quality indicators $\left(M_{i}\right)$ as estimates of the true quality indicators $\left(\mu_{i}\right)$ that are of interest:

$M_{i}=\mu_{i}+\epsilon_{i}$

where $\epsilon$ is the estimation error (which will tend to be larger for hospitals with smaller samples of patients). 
Our problem is how to use the entire vector of observed quality indicators for each hospital $\left(M_{i}\right)$ to best predict true quality differences across hospitals $\left(\mu_{i}\right)$. Conceptually, we can think of our problem as similar to that of minimizing the prediction errors $\left(v_{i}\right)$ in a regression of the form:

$\mu_{i}=M_{i} \beta_{i}+\nu_{i}$

where $\beta_{i}$ is a matrix of regression coefficients that provides the weights that should optimally be put on each quality indicator to predict most accurately the true quality differences across hospitals. One would expect these weights to vary by hospital because the precision of the observed quality indicators varies by hospital.

We cannot run a simple regression to estimate the weights in equation (5.2) because the true quality differences across hospitals are unobserved. Therefore, conventional provider report cards simply report the observed quality indicators as the best estimates of the true quality differences. This estimation is equivalent to assuming that each quality indicator has no error and, by itself, is the best predictor of the true quality differences-that is, the weight matrix in equation (5.2) $\left(\beta_{i}\right)$ has ones along the diagonal and zeros everywhere else.

But there are two problems with this approach. First, because the quality indicators $\left(M_{i}\right)$ are estimated with error, we can improve the mean squared error of the prediction by attenuating the coefficient toward zero. This attenuation should be greater for hospitals in which the quality indicators are not estimated precisely. This premise is the basic idea behind Bayesian shrinkage estimators (Morris 1983): the observed variation in quality indicators will tend to overstate the amount of actual variation across hospitals, so by pulling all the estimates (especially the more imprecise estimates) back toward the mean, we can improve prediction accuracy. The second problem with the conventional method is that it does not use any of the information available in other quality indicators. If the true quality differences across hospitals for other outcomes (e.g., other years, other patients) are correlated with the quality difference we are trying to predict, then using the information in estimates of these other quality indicators can improve prediction accuracy.

McClellan and Staiger (1999a) develop a simple method for creating estimates of $\mu$, based on equation (5.2), that incorporates both the shrinkage idea and information from all available quality indicators. 
The key to the solution is noting that estimating the optimal weights in equation (5.2) is analogous to estimating regression coefficients. The standard formula for estimating the regression coefficients in equation (5.2) is:

$$
\hat{\boldsymbol{\beta}}_{i}=\left[\operatorname{Var}\left(M_{i}\right)\right]^{-1} \operatorname{Cov}\left(M_{i}, \mu_{i}\right)=\left[\operatorname{Var}\left(\mu_{i}\right)+\operatorname{Var}\left(\epsilon_{i}\right)\right]^{-1} \operatorname{Var}\left(\mu_{i}\right)
$$

Thus, to form the optimal predictions of true quality differences across hospitals based on equation (5.2) one needs to estimate two matrices:

1. The variance-covariance matrix of the estimation error of the observed quality indicators $[\operatorname{Var}(\epsilon)]$. This matrix represents the precision in each quality indicator at each hospital. This matrix can be calculated in a straightforward manner, as is currently done when reporting the standard errors of conventional quality indicators.

2. The variance-covariance matrix of the true quality differences across hospitals $[\operatorname{Var}(\mu)]$. This matrix represents the correlation in true quality differences across measures and over time. To estimate this we note that $\operatorname{Var}(\mu)=\operatorname{Var}(M)-\operatorname{Var}(\epsilon)$. Thus, we can estimate the total variance in true quality differences across hospitals by subtracting the variance in the estimation error from the variance in the observed quality indicators.

Finally, we summarize the correlation across measures and over time with a time-series model (a vector auto-regression), which allows the construction of out-of-sample forecasts. This type of time-series model places little structure on the correlations and is commonly used as a flexible model for forecasting. For details of how this model is estimated and its empirical performance, see McClellan and Staiger (1999a).

To summarize, our method proceeds in three stages. First, we calculate a vector of conventional quality indicators for each hospital and for as many years as data are available. Second, we estimate the variance of the estimation error for the observed quality indicators $[\operatorname{Var}(\varepsilon)]$ and the variance of the true quality differences across hospitals [ $\operatorname{Var}(\mu)]$ as discussed above. Finally, we use these variance estimates to form predictions of true quality differences across hospitals based on equation (5.2) and using optimal weights derived from equation (5.3). We refer to estimates based on equation (5.2) as "filtered" estimates because these estimates seek to filter out the estimation error in the conventional quality indicators (and because our method is closely related to the idea of filtering in time series). 


\section{Comparing Hospitals Based on Outcomes for Eiderly Heart Attack Patients}

In this section, we summarize some of our recent work applying these methods to compare hospitals based on their quality of care for elderly heart attack patients (McClellan and Staiger 1999a, 1999b; Geppert, McClellan, and Staiger 2000). We begin by briefly describing the data. We then use three illustrative examples to demonstrate the value of our filtering method. First, we show that rankings based on the filtered measures are relatively stable over time and can forecast sizable differences across hospitals two to four years in the future. Second, we show that the filtered measures can be used to compare alternative quality indicators, choose among potential indicators, and evaluate the bias in measures that do not fully control for differences in patient severity and comorbidity across hospitals. Finally, we show that the filtered measures can be used to make meaningful comparisons across hospitals in a large metropolitan region.

\section{Data}

We use the same data as in McClellan and Staiger (1999a) for this analysis. Our hospital performance measures include serious outcomesmortality, and cardiac complications requiring rehospitalization-for all elderly Medicare beneficiaries hospitalized with new occurrences of acute myocardial infarction (AMI, or heart attacks) from 1984 through 1994, as well as for all elderly beneficiaries hospitalized for ischemic heart disease (IHD) from 1984 through 1994. To evaluate quality of care from the standpoint of a person in the community experiencing heart disease, we assign each patient to the hospital to which she was first admitted with that diagnosis. Our population includes over 200,000 AMI patients and over $350,000 \mathrm{IHD}$ patients per year. We limit our analysis of hospital performance to U.S. general short-term hospitals with at least two admissions in each year, a total of 3,954 hospitals that collectively treated over 92 percent of these patients.

For each AMI and IHD patient, our mortality measure is whether the patient died (for any reason) within seven, thirty or 365 days of admission. Mortality information includes all out-of-hospital deaths. Our complication measure is whether the patient was readmitted (at any hospital) between thirty and 365 days of admission for a cardiac-related diagnosis. For each hospital, we construct risk-adjusted 
outcome rates for each year and each diagnosis. These estimated hospital-specific intercepts are from a patient level regression (run separately by year, diagnosis, and outcome measure) that estimates the average outcome rate in each hospital, controlling for a fully interacted set of indicators for age, gender, black or nonblack race, and rural location. These estimates provide the conventional quality indicators on which our filtered estimates are based.

\section{Identifying High-Quality Hospitals}

As we saw in table 5.1, conventional quality indicators are unstable over time and are poor predictors of future hospital performance. In table 5.2, we show that this is not true when hospitals are ranked based on our filtered quality estimates. As in table 5.1, we focus on thirty-day AMI mortality as the outcome measure. Based on filtered estimates (using data available from 1984-1990), we identified the "best" and "worst" hospitals in 1990 as those in the top and bottom deciles in terms of thirty-day AMI mortality. The "best" hospitals are those with the best outcomes, those in the lowest decile of filtered mortality rates. Table 5.2 indicates how these best (column 1) and worst (column 2) hospitals performed two and four years later.

In contrast with conventional methods (see table 5.1), our filtering method can consistently identify best and worst hospitals and forecast future performance. Table 5.2 shows that, of the hospitals ranked in the best 10 percent by filtered measures in 1990, 52.1 percent are still ranked in the best 10 percent in 1992 and 42 percent are still in the best 10 percent in 1994. Almost none of these hospitals show up in the worst 10 percent in 1992 and 1994. The performance of hospitals we rank in the worst 10 percent in 1990 is similar, with 53.8 percent (43.4 percent) remaining in the worst 10 percent in 1992 (1994), and none showing up in the best 10 percent. Obviously, the difference between the results for conventional measures of thirty-day mortality (table 5.1) and the filtered measures (table 5.2) reflect the fact that conventional quality indicators in any given year are quite imprecise. As a result, the correlation in a hospital's ranking from one year to the next is quite low-below 0.2 between any two given years for conventional thirty-day AMI mortality rates. In contrast, filtered estimates of thirty-day AMI mortality rates do not change much over time: filtered estimates are correlated .96 between 1994 and 1992, and .89 between 1994 and 1990. 
Table 5.2

Comparisons of hospital rankings: 1990, 1992, and 1994

\begin{tabular}{|c|c|c|}
\hline & $\begin{array}{l}\text { Ranked in best } 10 \% \\
\text { (lowest mortality rate) } \\
\text { in } 1990\end{array}$ & $\begin{array}{l}\text { Ranked in worst } 10 \% \\
\text { (highest mortality rates) } \\
\text { in } 1990\end{array}$ \\
\hline \multicolumn{3}{|l|}{$\begin{array}{l}\text { Of the hospitals ranked as } \\
\text { indicated in 1990: }\end{array}$} \\
\hline \multicolumn{3}{|l|}{1992} \\
\hline $\begin{array}{l}\text { Percentage ranked in best 10\% } \\
\text { (lowest mortality rates) }\end{array}$ & $52.1 \%$ & $0 \%$ \\
\hline $\begin{array}{l}\text { Percentage ranked in worst } 10 \% \\
\text { (highest mortality rates) }\end{array}$ & $0.3 \%$ & $53.8 \%$ \\
\hline $\begin{array}{l}\text { Difference in mortality rate from } \\
\text { middle } 80 \% \text { of hospitals }\end{array}$ & -.037 & +.037 \\
\hline (Standard error) & $(.004)$ & $(.005)$ \\
\hline $\begin{array}{l}\text { Predicted difference in mortality } \\
\text { from middle } 80 \% \text { of hospitals, } \\
\text { based on } 1990 \text { forecast }\end{array}$ & -.038 & +.042 \\
\hline \multicolumn{3}{|l|}{1994} \\
\hline $\begin{array}{l}\text { Percentage ranked in best 10\% } \\
\text { (lowest mortality rates) }\end{array}$ & $42.0 \%$ & $0 \%$ \\
\hline $\begin{array}{l}\text { Percentage ranked in worst } 10 \% \\
\text { (highest mortality rates) }\end{array}$ & $0 \%$ & $43.4 \%$ \\
\hline $\begin{array}{l}\text { Difference in mortality rate from } \\
\text { middle } 80 \% \text { of hospitals }\end{array}$ & -.035 & +.036 \\
\hline (Standard error) & $(.004)$ & $(.006)$ \\
\hline $\begin{array}{l}\text { Predicted difference in mortality } \\
\text { from middle } 80 \% \text { of hospitals, } \\
\text { based on } 1990 \text { forecast }\end{array}$ & -.029 & +.032 \\
\hline
\end{tabular}

Note: Rankings based on filtering methods. Outcome measure: Filtered risk-adjusted thirty-day AMI mortality.

Even more important, mortality rates in 1992 and 1994 for the hospitals ranked in the best and worst 10 percent in 1990 are significantly different from the remaining 80 percent of hospitals (see table 5.2). Hospitals ranked in the best 10 percent in 1990 had, on average, 3.8 percentage points lower mortality in 1992 , and 3.5 percentage points lower mortality in 1994, relative to the middle 80 percent of hospitals. Conversely, hospitals ranked in the worst 10 percent in 1990 had 3.7 percentage points higher mortality in 1992, and 3.6 percentage points higher mortality in 1994, relative to the middle 80 percent of hospitals. These differences in mortality are large relative to the average thirty-day mortality rate for AMI patients of about 19 percent in 1994. They imply that 
the hospitals we rank in the best 10 percent using filtered data in 1990 had a mortality rate of 15.5 percent in 1994, while the hospitals ranked in the worst 10 percent in 1990 had a mortality rate of 22.6 percent in 1994. In fact, forecasts derived from the filtering methodology, based only on data available through 1990, accurately predicted these differences in 1992 and 1994 as seen in the fourth and eighth rows of table 5.2. Thus, hospital rankings based on the filtered estimates appear to identify large and persistent differences across hospitals in patient outcomes.

\section{Comparing Alternative Quality Indicators}

Is thirty-day AMI mortality the right quality indicator to focus on? Would hospital rankings change if one controlled for additional (but costly) information on differences in patient severity and comorbidity across hospitals? Ideally we would like to limit the number of quality indicators being collected and reported, both to reduce data collection costs and to simplify reporting. One method of doing so would be to compare hospital rankings across quality indicators: if two quality indicators produce similar rankings, then one of them (the more expensive, or the one that identifies smaller differences across hospitals) could be eliminated. The imprecision found in conventional quality indicators makes such a comparison difficult because it can generate a low correlation between any two quality indicators (as we saw in comparisons from one year to the next). By reducing the imprecision found in conventional quality indicators, filtered measures can be used more easily to compare alternative quality indicators, choose among potential indicators, and evaluate the bias in measures that do not fully control for differences in patient severity and comorbidity across hospitals.

Table 5.3 contains estimates from 1994 of the relationship between various quality indicators and thirty-day AMI mortality. The first two columns are based on filtered measures, and the second two columns are based on conventional estimates (unfiltered). For both filtered and conventional estimates, we report the standard deviation of each quality indicator across hospitals (a measure of the variation in the estimated outcome rate across hospitals) and the correlation of each indicator with thirty-day AMI mortality.

We consider various alternative quality indicators. First, we consider an alternative measure of thirty-day AMI mortality that controls for a 
Table 5.3

Comparisons across alternative outcome measures (filtered versus conventional estimates)

\begin{tabular}{|c|c|c|c|c|}
\hline & \multicolumn{2}{|l|}{ Filtered estimates } & \multicolumn{2}{|c|}{ Conventional estimates } \\
\hline & $\begin{array}{l}\text { Standard } \\
\text { deviation } \\
\text { across hospitals }\end{array}$ & $\begin{array}{l}\text { Correlation } \\
\text { with 30-day } \\
\text { AMI mortality }\end{array}$ & $\begin{array}{l}\text { Standard } \\
\text { deviation } \\
\text { across hospitals }\end{array}$ & $\begin{array}{l}\text { Correlation } \\
\text { with 30-day } \\
\text { AMI mortality }\end{array}$ \\
\hline $\begin{array}{l}\text { 30-day AMI } \\
\text { mortality with } \\
\text { detailed risk } \\
\text { adjustment }^{\mathrm{a}}\end{array}$ & .029 & .91 & .096 & .91 \\
\hline $\begin{array}{l}\text { 7-day AMI } \\
\text { mortality }\end{array}$ & .024 & .99 & .079 & .82 \\
\hline $\begin{array}{l}\text { 30-day AMI } \\
\text { mortality }\end{array}$ & .028 & 1 & .095 & 1 \\
\hline $\begin{array}{l}\text { 1-year AMI } \\
\text { mortality }\end{array}$ & .031 & .95 & .107 & .75 \\
\hline $\begin{array}{l}\text { Mortality or } \\
\text { cardiac readmit } \\
\text { within } 1 \text { year }\end{array}$ & .032 & .72 & .109 & .56 \\
\hline $\begin{array}{l}\text { 1-year IHD } \\
\text { mortality }\end{array}$ & .014 & .62 & .065 & .04 \\
\hline
\end{tabular}

${ }^{\text {a Comparison of thirty-day AMI mortality adjusted for patient demographics with }}$ thirty-day AMI mortality adjusted for detailed patient risk factors is based on data from Health Care Financing Administration's (HCFA's) cooperative cardiovascular project. All other comparisons are based on measures derived from Medicare claims data as described in the article.

detailed set of patient severity and comorbidity variables developed from comprehensive chart reviews; these patient data are far more detailed than the information available in claims. These data, which were developed for the hospitals in our study through HCFA's Cooperative Cardiovascular Project, can provide evidence on whether rankings based on claims data are biased by the lack of detailed risk adjusters. We also compare thirty-day mortality to seven-day and one-year mortality for AMI patients. The correct amount of follow-up time is unclear, and shorter-term mortality is generally cheaper to collect. In a third comparison, we consider a measure capturing a broader range of adverse outcomes for AMI: either death or readmission for a cardiac diagnosis within one year. We do not count readmissions in the first thirty days as adverse events because many of these are for appropriate follow-up care, e.g., bypass surgery. Finally, we consider a quality indi- 
cator for an entirely different sample of patients: one-year mortality for patients with ischemic heart disease (IHD). Like AMI, IHD is a cardiac condition and one would expect quality of care to be positively correlated across these two closely related medical conditions.

Two facts are apparent from table 5.3. First, the filtered measures vary considerably across hospital (column 1), with a standard deviation of around 3 percentage points for the AMI measures (seven-day, thirty-day and one-year mortality, and one-year mortality or readmit), and 1.4 percentage points for one-year IHD mortality. Conventional estimates are considerably more variable for every quality indicator (column 3), but most of this variation is a result of the noise in conventional estimates.

The second fact apparent from table 5.3 is that many of the filtered measures are highly correlated. Most important, we find that the filtered thirty-day AMI mortality rate (which controls only for differences across hospitals in a limited set of patient demographics) is highly correlated (0.91) with a filtered thirty-day AMI mortality rate based on detailed risk adjustment. In other words, filtered AMI mortality rates, based on limited risk-adjustment using only the patient variables commonly available in claims data, generate essentially the same rankings as measures based on more detailed risk adjustment that require more extensive data collection. Thus, for this example, there appears to be little bias in hospital rankings that rely on AMI mortality measures derived from claims data.

Filtered thirty-day AMI mortality rates are even more highly correlated (over 0.95) with seven-day and one-year mortality. In other words, filtered short-term (even seven-day) mortality rates generate essentially the same rankings as long-term mortality measures that require more extensive patient follow-up. Thus, at least for AMI admissions, there appears to be little reason to incur the extra costs and delays involved in collecting long-term mortality information.

Finally, the correlation of filtered thirty-day mortality with the remaining measures (AMI mortality or cardiac readmission within one year, one-year IHD mortality) is less strong but still above 0.6. While these quality indicators are related to AMI mortality, they do seem to capture independent information on hospital quality along other dimensions. Thus, these two measures may provide useful additional information for comparing hospitals.

Our conclusions would have been quite different if we had tried to do a similar analysis using conventional estimates of these quality indi- 
cators. Because these conventional estimates are based on small samples from a single year, they are imprecise. As a result, they tend to understate considerably the correlation between thirty-day AMI mortality and the alternative measures, especially when making comparisons to a measure from a different sample of patients (e.g., one-year IHD mortality) because the estimation error in such measures is uncorrelated. For other measures (e.g., thirty-day AMI mortality with detailed risk adjustment) the conventional estimates remain highly correlated because the noise in the two measures is highly correlated-e.g., a chance death for a few average patients will adversely affect both measures.

Overall, this example illustrates how comparisons across filtered quality measures can be used to identify a subset of core quality indicators. This example provides an empirical basis on which to eliminate redundant measures (such as long-term mortality). It also provides an empirical basis on which to explore the potential bias in claims-based measures.

\section{Comparing Hospitals in a Metropolitan Market}

One of the goals in developing quality indicators is to allow meaningful comparisons to be made across hospitals in a given region or market. Thus, from a practical standpoint, quality indicators must be precise enough to allow one to identify consistently the best (and worst) hospitals in a market. The filtered estimates for thirty-day AMI mortality are quite precise relative to conventional quality indicators and appear to be quite useful for comparing hospitals within markets (for example, see McClellan and Staiger 1999b).

To illustrate this point, figure 5.1 presents plots of filtered and conventional estimates of thirty-day AMI mortality rates for 1994 (relative to the national average) for twenty-six hospitals ranging in size from fifteen to $175 \mathrm{AMI}$ admissions and located in a large metropolitan area. In this figure, hospitals are ordered from left to right according to how they rank among the twenty-six hospitals in terms of the filtered (bottom panel) and conventional (top panel) measures. The vertical axis gives the estimated mortality rate (relative to the national average), with a value of -0.1 , for example, indicating that mortality at the hospital was ten percentage points below the national average. Confidence bars indicate a 1.4 standard error confidence interval around each hospital's estimate, so that hospitals with nonoverlapping confidence in- 


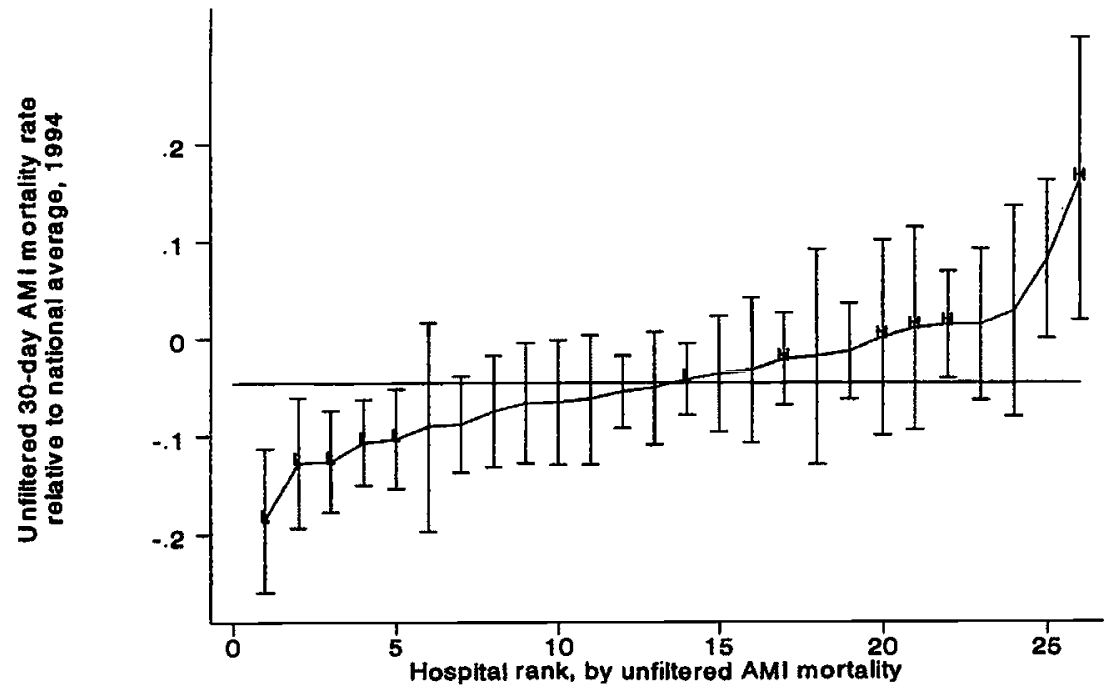

A. Unfiltered 30-day AMI mortality rates, 1994

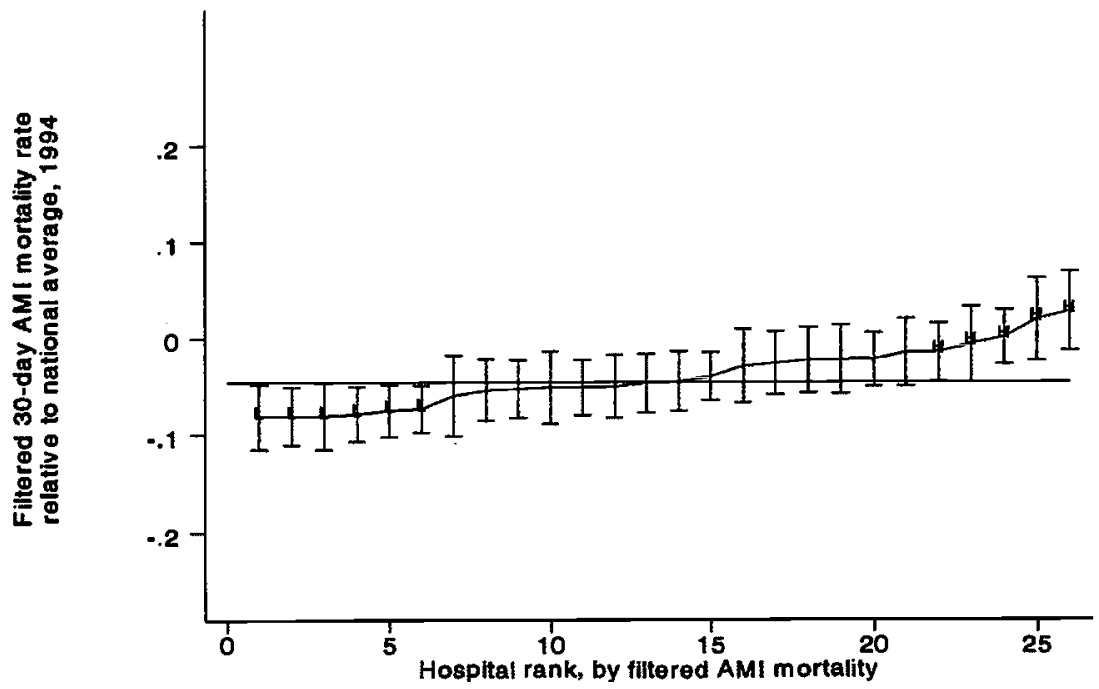

\section{B. Filtered 30-day AMI mortality rates}

\section{Figure 5.1}

Comparison of unfiltered (panel A) and filtered (panel B) 30-day AMI mortality rates at hospitals in a large metropolitan region, 1994

Absolute difference between each hospital's mortality rate and the national average in 1994, adjusted for patient demographics. Error bars provide a 1.4 SE confidence interval, so that hospitals with nonoverlapping intervals are statistically different at the $5 \%$ level. The horizontal line marks the median mortality rate in the region. Hospitals labeled " $\mathrm{H}^{\prime \prime}$ and " $\mathrm{L}$ " were identified as high and low mortality hospitals, respectively, based on filtered mortality rates in 1994. 
tervals are statistically different at the 5 percent level. In each panel, we have drawn a horizontal line through the median mortality rate in the area as a reference point.

It is apparent that the filtered estimates (bottom panel) are much more precise than are the conventional estimates (top panel). The confidence intervals on the filtered estimates are roughly half the size of the intervals on the conventional estimates, with the improvement in precision much greater for the smallest hospitals. ${ }^{1}$ The extreme estimates apparent in the conventional measures (a few hospitals with a mortality more than fifteen percentage points away from the median) are not present in the filtered estimates. Nonetheless, the filtered estimates identify large differences in mortality rates: six hospitals (marked " $\mathrm{L}$ " in figure 5.1) have filtered confidence intervals lying entirely below the median, while another five hospitals (marked " $\mathrm{H}$ " in figure 5.1) have filtered confidence intervals lying entirely above the median. The difference in average estimated mortality rates between hospitals in these low and high mortality groups is over eight percentage points.

Another way to demonstrate the ability of the filtered estimates to identify differences consistently across hospitals is to plot the measures over time. Figure 5.2 plots conventional estimates of thirty-day AMI mortality rates for each hospital in the region between 1984 and 1994. To reduce clutter, we have broken the sample into four roughly equal groups based on the average annual number of AMI admissions at each hospital over this time period, and we plot the data for each group separately. For comparison, we continue to label those hospitals identified by the filtered measures as having high $(\mathrm{H})$ and low $(\mathrm{L})$ mortality in 1994.

The imprecision in conventional estimates is immediately apparent from figure 5.2. The conventional mortality estimates change dramatically from year to year and follow no apparent pattern. As a result, it is nearly impossible to identify hospitals that have consistently low or high mortality, and equally impossible to discern any trends in these measures over time.

In contrast, the same plot using filtered data (figure 5.3) can identify clearly the differences across hospitals and over time. Hospitals that were ranked as having high $(\mathrm{H})$ mortality in 1994 have consistently had high mortality over this period, although some showed more improvement over time than others. Similarly, hospitals that were ranked as having low mortality in 1994 tended to be larger hospitals and to 

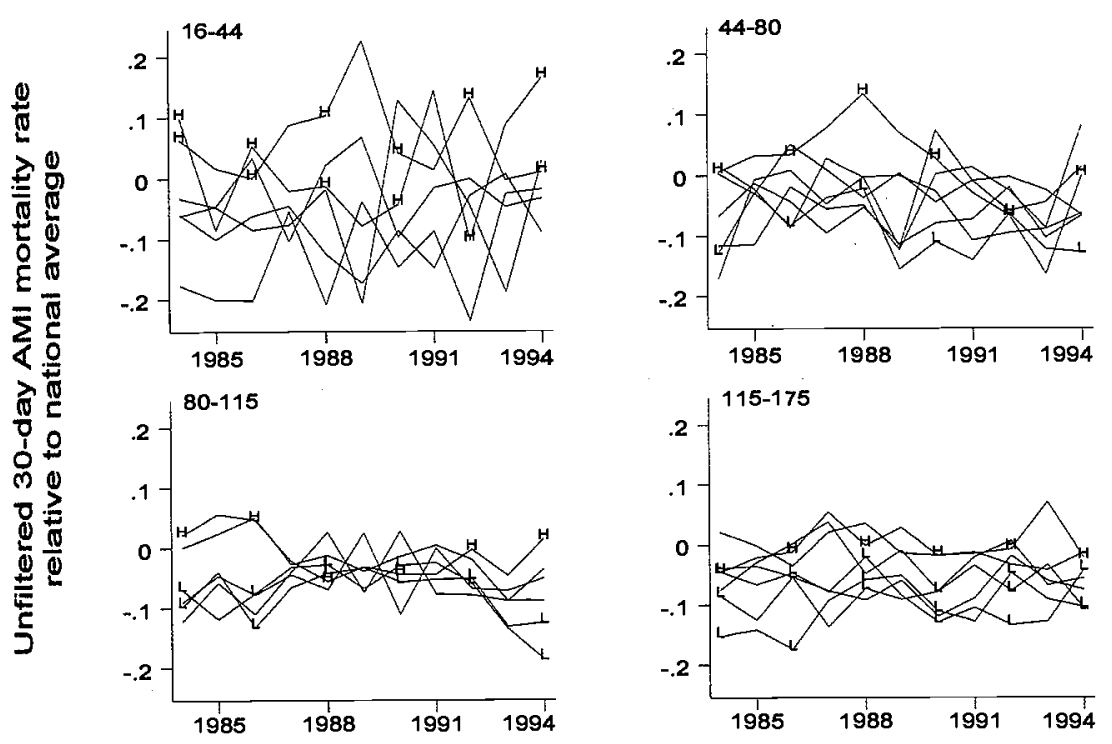

\section{Year}

\section{Figure 5.2}

Comparison of unfiltered 30-day AMI mortality rates at hospitals in a large metropolitan region, 1984-1994

Plotted separately by average annual AMI volume: 16-44 admissions per year (upper left), 44-80 admissions per year (upper right), 80-115 admissions per year (lower left), and 115-175 admissions per year (lower right). The vertical axis gives the absolute difference between each hospital's mortality rate and the national average in that year, adjusted for patient demographics. Hospitals labeled " $\mathrm{H}^{\prime \prime}$ and " $\mathrm{L}$ " were identified as high and low mortality hospitals, respectively, based on filtered mortality rates in 1994.

have low mortality throughout the period. Among the largest hospitals (115 to $175 \mathrm{AMI}$ admissions), the filtered estimates can identify changes in the relative rankings among the three best hospitals in the region. Thus, this example suggests that the filtered measures can be used to make meaningful comparisons across hospitals and over time within a large metropolitan region.

\section{Implications}

This article provides a brief overview of how our method for extracting the signal from many noisy quality measures can support better evaluations of the quality of health care providers. The simple examples pre- 

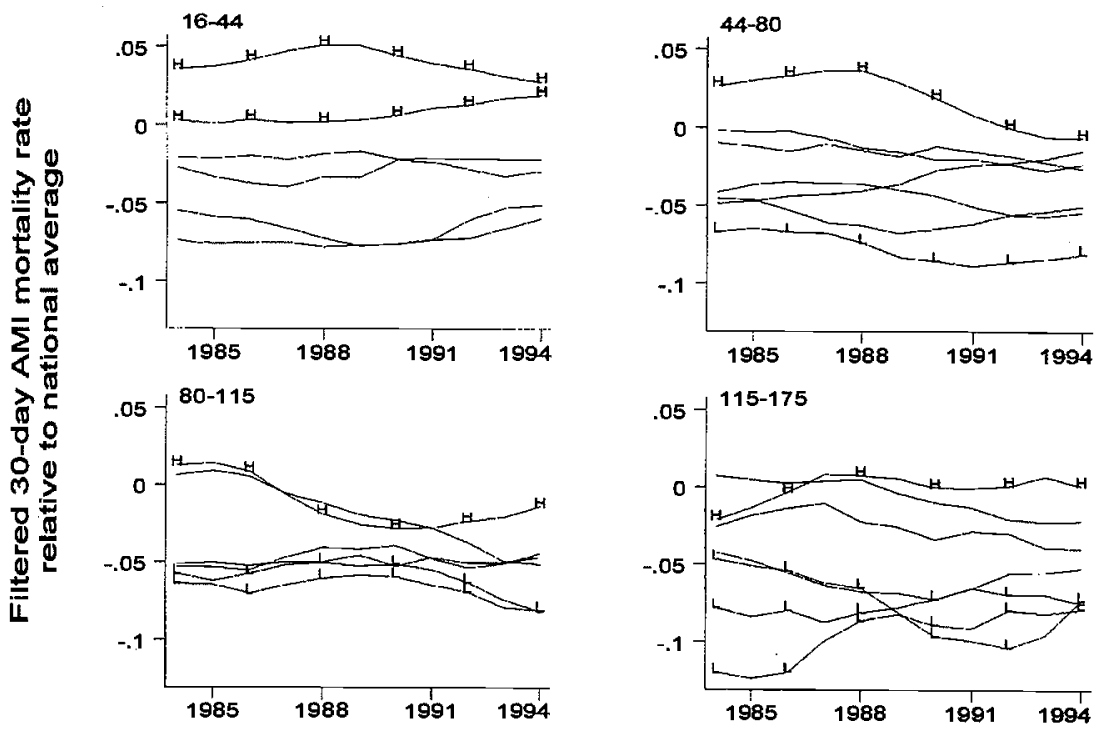

\section{Year}

\section{Figure 5.3}

Comparison of filtered 30-day AMI mortality rates at hospitals in a large metropolitan region, 1984-1994

Plotted separately by average annual AMI volume: 16-44 admissions per year (upper left), 44-80 admissions per year (upper right), 80-115 admissions per year (lower left), and 115-175 admissions per year (lower right). The vertical axis gives the absolute difference between each hospital's mortality rate and the national average in that year, adjusted for patient demographics. Hospitals labeled " $\mathrm{H}$ " and " $\mathrm{L}$ " were identified as high and low mortality hospitals, respectively, based on filtered mortality rates in 1994.

sented here illustrate how the method may offer an integrated solution to the key problems that limit the usefulness of currently available quality indicators. Compared to existing methods for assessing the quality of health care providers, our approach has several advantages. It limits measurement costs by providing a sound empirical basis for comparing and choosing among alternative quality indicators. More important, our method performs far better than alternative approaches for eliminating the noise problem that has plagued efforts to develop and apply practical quality indicators.

We are applying these methods to a larger range of health problems, patient populations, and provider networks and integrating them into quality improvement programs. These efforts require collaboration 
among analysts, health plans, providers, purchasing groups, and government agencies to collect and integrate relevant information for constructing the quality measures and using them to help guide decision making and improve provider quality.

One feature of our collaborative work is the use of meaningful quality measures that would be difficult or impossible for individual participants in the collaboration to obtain. For example, the most important performance measures for serious health conditions like heart disease and cancer involve long-term outcomes (e.g., two-year mortality), which are costly or impossible for providers and health insurers to collect. In contrast, government agencies commonly collect mortality and disease surveillance data needed for constructing such measures, but they may not have information on the responsible medical groups or physicians for the patients involved. Through the participation of all of these groups, constructing measures of quality for serious health conditions becomes feasible.

Another feature of our approach is the optimization of the use of detailed but costly clinical data to account for patient characteristics that may result in biased measures of quality. As we illustrated here, we include some measures based on clinically detailed risk adjustment (e.g., with data drawn from chart abstracts) for some of the patients in our analysis to evaluate potentially important differences across providers in patient severity of illness. Using these gold standard measures, we can identify valid proxy measures that are less costly and more readily available or adjust these measures appropriately. As a result, we can extract more reliable data about true provider quality from the lower cost measures. These steps minimize the overall resource burden of our data collection and measure development and improve their validity.

A third component of our collaboration involves integrating our new outcome-based measures with those developed in established continuous quality improvement initiatives, for example, the standardized measures of patient satisfaction and process of care developed by the National Committee for Quality Assurance (NCQA). These measures have been tested and applied extensively, but their relationship with important outcomes of care is not well understood. We are investigating the relationship between performance in these dimensions of quality and important outcomes of care to determine the extent to which these readily available measures can substitute for more comprehensive outcome evaluations.

The most important goal for many of our collaborators is to use these measures to improve quality of care. First, we are identifying the struc- 
tural features and processes of care that are related most closely to important outcomes and thus identifying specific practice changes that are most likely to improve provider quality. Our methods provide empirical evidence of the magnitude and direction of relationships between these modifiable factors and outcomes. Such evidence can be used to ensure that continuous quality initiatives focus on identifying and changing provider practices where there is the greatest opportunity for improving outcomes. Second, our methods can be used to obtain much more precise estimates of the impact of interventions such as provider training or patient education.

Through all of these steps, our collaborative approach to quality evaluation and improvement is developing performance measures that are precise and reliable and therefore more acceptable to providers and payers. This undertaking is complex and long term, but our results to date suggest that the potential for evidence-based quality improvement in health care is much greater than many experts now believe.

\section{Notes}

Prepared for the NBER conference on Frontiers in Health Policy Research, June 17, 1999, Washington DC. This article draws heavily on our own ongoing work and work with various collaborators. The National Institute of Aging, The Health Care Financing Administration, and the Olin Foundation provided financial support at various times for this work. We thank Jon Skinner and Jeff Geppert for helpful comments on an earlier draft. We also thank our collaborators, including Jeffrey Geppert, Jeffrey Horbar, Thomas Kane, Haruko Noguchi, Jeannette Rogowski, and Yu-Chu Shen.

1. Note that one cannot make direct comparison across the two panels because hospitals are ranked differently by the two measures.

\section{References}

Bindman, A. B., 1999. "Can Physician Profiles Be Trusted?" JAMA 281(22): 2142-2143.

Dranove, D., and M. A. Satterthwaite, 1992. "Monopolistic Competition When Price and Quality Are Imperfectly Observable." The RAND Journal of Economics 23(4): 518-534.

Enthoven, A., and R. Kronick, 1989. "A Consumer-Choice Health Plan for the 1990s: Universal Health Insurance in a System Designed to Promote Quality and Economy." New England Journal of Medicine 320(1): 29-37 (part 1) and 320(2): 94-101 (part 2).

Geppert, J., M. McClellan, and D. Staiger, 2000. "Hospital Quality, Treatment Effects, and Health Outcomes: Analyzing Differences in Medical Practices." Manuscript, February 2000.

Hofer, T. P., R. A. Hayward, S. Greenfield, E. H. Wagner, S. H. Kaplan, and W. G. Manning, 1999. "The Unreliability of Individual Physician 'Report Cards' for Assessing the Costs and Quality of Care of a Chronic Disease." JAMA 281(22): 2098-2105. 
Iezzoni, L., 1997. Risk Adjustment for Measuring Healthcare Outcomes. Chicago: Health Administration Press.

Institute of Medicine (IOM), 1990. Medicare: A Strategy for Quality Assurance, I, Washington, DC: National Academy Press.

Krakauer, H., R. C. Bailey, K. J. Skellan, et al., 1992. "Evaluation of the HCFA Model for the Analysis of Mortality Following Hospitalization." Health Services Research 27(3): 317-335.

Landon, B., L. I. Iezzoni, A. S. Ash, et al., 1996. "Judging Hospitals by Severity-Adjusted Mortality Rates: The Case of CABG Surgery." Inquiry 33(2): 155-166.

McClellan, M., and D. Staiger, 1999a. "The Quality of Health Care Providers." National Bureau of Economic Research working paper.

McClellan, M., and D. Staiger, 1999b. "Comparing Hospital Quality at For-Profit and Not-for-Profit Hospitals." in D. Cutler (ed.), The Changing Hospital Industry: Comparing Not-for-Profit and For-Profit Institutions, Chicago: University of Chicago Press, 2000, pp. 93-112.

Medicine E Health, 1997. "Gauging Quality Regulation's Impact on Premium Costs," 51:1.

Morris, C., 1983. "Parametric Empirical Bayes Inference: Theory and Applications." JASA 78(381): 47-55.

Park, R. E., R. H. Brook, J. Kosecoff, et al., 1990. "Explaining Variations in Hospital Death Rates: Randomness, Severity of Illness, Quality of Care." JAMA 264(4): 484-90.

Schneider, E. C., and A. M. Epstein, 1998. "Use of Public Performance Reports: A Survey of Patients Undergoing Cardiac Surgery." JAMA 279(20): 1638-42. 